\title{
Evolution and Prospect of Chinese Martial Arts during the Rising Course of China
}

\author{
QiaoYaJun \\ Zhengzhou shengda college of economic and trade Zhengzhou 451191
}

Key words: martial arts, evolution, prospect

\begin{abstract}
With a strategic view of historical materialism and specialized way of thinking, the essay makes a dialectical demonstration of the origin and evolution of sports, traditional and school martial arts, martial arts school, social and movie martial arts and its ranking system and so on. The study shows that: Like any other culture, Chinese martial art is a dynamic cultural system evolving with progressive society toward diversification. It has a bright prospect in the future and may be widely used and of great significance.
\end{abstract}

\section{Introduction}

The origin and future of martial arts has been always a concern for Chinese people. In retrospect, the evolution of Chinese Martial Arts (simplified as martial arts hereafter) is closely related to its social progress. And it has had a definite aim since its foundation, that is, for the time and for the people. During the prolonged age of cold weapons, though martial arts and military affairs were incomparable, they derived from the same source and origin. While warrior were involved in combat in ancient time, martial arts, together with military affairs, served as a strong support for power as well as hope and guarantee of ordinary people's well-being. As wise men shall fulfill his intelligence, martial arts__ a life culture focused on fighting skills_— has been well retained and passed to modern time. Adding further enhancement and development, it grows into effective means for physical fitness, appreciation and entertainment surprisingly. Culture is diversifying over time, which is a law of development. Three decades ago, martial art was described merely as a fight, which nowadays features obviously diversified development trend. In whatever cultural arena, forecast is a critical subject while there is a heated dispute over its reform. Despite disputes over its type, the main frame or contents involved in those are hardly differentiated. Thus, this passage will base on the reality and prospect of martial arts and make a generalization from such main aspects as sports, traditional and school martial arts, martial arts school, social and movie martial arts and its ranking system and so on.

\section{Competitive Martial Arts}

Competitive martial arts routine is highly valued. In Nation Games which is held every four years, it is an item of multiple gold medals. As it is oriented at extreme challenges to people's physical potential, strength and skills, sportsmen is trained toward fighting for medals, with their excellent performance as a goal for instructors. For the public, it is an enjoyment of beauty and happiness. While for sportsmen, its main function consists in demonstrating physical capability and charm of martial arts instead of fitness. Consequently, competitive martial arts forms will definitely choose sophisticated, difficult, elegant, new and authentic skills as its orientated value.

\section{Traditional Martial Arts}

Such a saying did not exist until 1970s when it generally referred to all forms of martial arts. While competitive martial arts system gradually forms with the time, traditional one is distinguished from it as such. Traditional martial arts is national sports founded on traditional Chinese culture, with attack and defense as main contents and routines and free fighting as main forms. It lays great emphasis on conditioning and meditation exercises as well as internal and external training. 
Traditional martial art is the original form of Chinese martial arts. It is also the source of other types. Its origin, evolvement, distinct characteristics and systematic contents are of overwhelming appeal to ordinary people.

\section{School Martial Arts and Martial Arts Schools}

Generally speaking, school martial art is for all school students to exercise and entertain in spare time or $\mathrm{PE}$ classes for fitness purposes. It mainly features the same arrangement of lessons by leading educational departments with identical and unified textbooks. In some senses, as China is the origin of martial arts, relevant activities should be developed in all schools uniquely. Nevertheless, it is on the contrary side. Chinese students (elementary and middle school students in particular) have so long been influenced by exam-oriented educational system that they emphasize theoretical practices rather than practical sports exercises. Such trend is still to be reversed. On the other side, bringing martial arts to schools and classes has been advocated for years, but its effect is poor and feeble. Martial arts are introduced in elementary, middle schools and colleges to a limited extent. At the same time, school martial arts are largely interfered by conventional ideas and misconceptions together with shocks from western sports. Nowadays, it is not that the majority teenagers are either persistent or callous to martial arts, but that there is no suitable conditions and atmosphere for them to truly experience its actual function. Their idols mostly are heroes from martial arts fiction or supermen in films. They don't like the motto of "hard training for mastering".

\section{Social Martial Arts}

Apart from martial arts' multiple functions, the main reason why it is favored is that it comes among folk people and serves for them. The number of people involving in such exercises is a major benchmark for its popularity. Today a simple terminology can no longer explain precisely the connotation of martial arts. Social martial arts basically belong to the whole system. It is getting thought-provoking as China gains more strength, people's living standard is constantly raising and more attentions are paid to physical health. Narrowly speaking, social martial arts are distinguished from both competitive and traditional ones. Comparatively, it is a type of exercises ordinary people do out of interest or need for entertainment. Its main features are: 1) People who exercise are from all walks of life. They use spare time or place to do individually or collectively; 2) Items for exercising vary with each person. There are training of stance, forms, single movement, integrated moves, breathing and various weapons. All movements are simple and easy for exercises, without restraint of skills; 3) Exercises are done mostly in the morning or at night; 4) Laid-off or retired people account for a large part. Martial arts enthusiasts often adapt and restructure on the basis of original forms, which has a great potential for growth. Social martial arts are of abundant connotations and strong inclusiveness. Mixed fitness items in domestic and foreign countries as well as in different ethnic groups will bear substantial impact on development of social martial arts in the future.

\section{Movie Martial Arts}

Martial arts raise the audience rating for media, which conversely enables its effective transmission and publicity. Such combination for mutual benefits is great potential and excellent prospect. With the development of society, people are pursuing better life as well as upgrading abilities for cultural appreciation. For ordinary martial arts are not as appealing as before, reform and innovation is required for wonderful and thrilling effect as well as for its further development. Under such circumstances, all included in Chinese martial arts could be displayed and applied to movies, like drama, dance, acrobatics, sketch, hard Qigong and stunt and skills in the system.

\section{Ranking Systems}

At the end of 1980s, when competitive martial arts routines reached maturity, national functional departments and people in martial arts field started to think about formulating a system ranking skills of martial arts talents. After several round of talks and discussions, they decided to set 
three honor levels as first-grade sportsman, masters and learners for participants of competitive martial arts. Afterwards, ranking of social and traditional martial arts became an urgent problem because it entailed in a group of martial arts practitioners with the most space and population. As a result, basing on suggestions from experts and actual requirements for future expansion of martial arts, relevant departments referred to successful experiences on ranking of karate, judo and taekwondo and finally established its own ranking system in 1997. A whole set of standards for skill assessment is included in the system. In 1998, the state formally carried out assessment of the ranking system. As surveyed, an estimated 125,000 members ranking as junior, medium and senior (1 9) have registered up to now in martial arts management center of State General Administration of Sports. Averagely, only about 10,000 will take part in the system annually, which is somewhat far from original intentions. The main reason is insufficient experiences, attention and publicity. Most practitioners don't know its existence or about how to join in otherwise without matching textbooks or convenient procedures. After the 2008 Beijing Olympic Games, efforts were enhanced to coordinate its comprehensive development, with ranking work gaining more attention. In order to make it more systematic and scientific, the ranking textbook adhered to a combination of theory and practices, in which experts elaborated at length historical reform, styles and features and principal skills of more than ten traditional fist types, especially skills of that from $1^{\text {st }}$ to $6^{\text {th }}$ rank. While striving to retain properties of various fist types, the textbook centered on easy learning, practicability and acquiring progress, highlighting technological trait of traditional martial arts. Routines adapted in the book are fit for both solo and pair training. Adding supplementary CDs and videos, it has an effect of "returning to nature". Ranking system is the most basic content for learning, targeting at beginners. With publication and spread of textbooks on ranking system, it will be of maximum benefit to people engaged in social and school martial arts as well as in international fields. Looking into the future, ranking system will play a major role in publicity of martial arts at home and abroad.

\section{References}

[1] Anthony Giddens. Sociology. Translated by Zhao Xudong. [M].Bejing: Peking University Press, 2007: 137.

[2] Chen Xin. Chen-style Tai chi Illustration. [M].Taiyuan: Shanxi Science and Technology Publishing House (SSTP), 2006: 12.

[3] Li Shengfu. Development Strategy of Chinese Martial Arts after 2008 Beijing Olympic Games. [J].Sports Science, 2008, 28(9): 85. 